\title{
Polymorphism in repeated 16S rRNA genes is a common property of type strains and environmental isolates of the genus Vibrio
}

\author{
Claudia Moreno, Jaime Romero and Romilio T. Espejo
}

Laboratorio de

Bioingeniería, Instituto de Nutrición y Tecnología de los Alimentos, Universidad de Chile, Macul 5540, Santiago, Chile
Author for correspondence: Romilio T. Espejo. Tel: +562 6781426. Fax: +5622214030. e-mail:respejo@uec.inta.uchile.cl

\begin{abstract}
Analysis of the 165 rDNAs obtained from cultures of single colonies of either type collection strains or environmental strains of the genus Vibrio revealed the presence of polymorphism in every one of the strains examined. Polymorphism was detected by visualization of heteroduplexes produced after 165 rDNA PCR amplification, a procedure that allows for the screening of a large number of isolates. Amplified 16S rDNAs obtained from both Vibrio parahaemolyticus and an environmental strain were cloned. Their nucleotide sequences revealed differences of up to $2 \%$ among 165 rDNAs from the same strain. Polymorphic sites were concentrated in a recognized variable stem-loop of bacterial 165 rRNA that contained in some cases up to $83 \%$ of the total mismatches observed. Most of the substitutions present in the stem-loop region showed compensating base covariation. The accumulation of so many compensating changes in the stem-loop region implies that the divergence of the different versions of this stem-loop is relatively ancient. This divergence could be the result of either a selection process or a lateral transfer of independently evolved genes.
\end{abstract}

Keywords: marine, $16 \mathrm{~S}$ rDNA, rrn

\section{INTRODUCTION}

Acceptance of $16 \mathrm{~S}$ rRNA as an appropriate tool for the reconstruction of evolutionary history and phylogenetic relationships (Amann et al., 1995; Gray et al., 1984) has been based on the fact that, among other assumptions, multiple copies of rRNA genes present in a single organism containing more than one $r r n$ operon are either identical or nearly identical in nucleotide sequence. This assumption seems to be valid for some bacterial species, but not for others. For example, among some bacterial strains, for which the whole genome sequence is available, there are some strains with multiple $r r n$ operons that nevertheless show no intra$16 \mathrm{~S}$ rDNA polymorphism. Examples of these are Treponema pallidum, Xylella fastidiosa, Synechocystis PCC 6803 with two rrn operons; Campylobacter jejuni with three, Neisseria meningitidis with four and Haemophilus influenzae with six rrn operons. On the other

The GenBank accession numbers for the sequences reported in this paper are AF388386 (Vp23), AF388387 (Vp16), AF388388 (F44), AF388389 (Vp27), AF388390 (F6), AF388391 (3d2), AF388392 (3d4), AF388393 (3d7) and AF388394 (3d8). hand, Helicobacter pylori and Ureaplasma urealyticum with two, Escherichia coli with seven, Vibrio cholerae with eight and Bacillus subtilis with ten rrn operons show differences between $16 \mathrm{~S}$ rDNAs in different operons ranging from 0.6 up to $2 \%$ (www.ncbi.nlm. nih.gov and www.tigr.org). Polymorphism in repeated $16 \mathrm{~S}$ rRNA genes had been initially suggested by the observation of nucleotide variations from 1 to $5 \%$ between pairs of $16 \mathrm{~S}$ rRNA sequences from one bacterial strain or different bacterial strains of the same species deposited in GenBank (Felsenstein, 1985). 16S rRNA sequences from bacterial strains deposited in databases are usually obtained from a clone of a single operon or from the bulk of either $16 \mathrm{~S}$ rDNA or $16 \mathrm{~S}$ rRNA. Cloning of a single operon results in a sequence that may differ from those of the other operons, while direct sequencing of PCR or RT-PCR products produces a mean sequence in which differences between operons may be hidden. $16 \mathrm{~S}$ rDNA polymorphism has been previously observed in E. coli strain PK3 (Cilia et al., 1996), Paenibacillus polymyxa (Nubel et al., 1996), Mycobacterium celatum (Reischl et al., 1998), Thermobispora bispora (Wang et al., 1997), Streptomyces strains (Ueda et al., 1999) and in Thermomonospora chromogena (Yap et al., 1999). 
More recently, Dahllof et al. (2000) showed 16S rDNA intraspecies heterogeneity in different bacterial isolates by denaturing gradient gel electrophoresis that was not observed in the gene for the RNA polymerase $\beta$ subunit $(r p o B)$. Altogether these data indicate that the existence of operons with different $16 \mathrm{~S}$ rRNA in the same organism is a proven, though not general, phenomenon. It remains to be shown how common it is in nature.

The genus Vibrio contains a large number of closely related bacterial species with $16 \mathrm{~S}$ rRNAs differing in nucleotide sequence from less than $1 \%$ up to $6 \%$ (Dorsch et al., 1992; Ruimy et al., 1994). The presence of several $r r n$ operons has been shown in some Vibrio spp. and it may be common among members of this genus (Yamaichi et al., 1999). Vibrio fischeri and Vibrio harveyi contain 8-11 operons (Lamfrom et al., 1978; Wolfe \& Haywood, 1993; Fegatella et al., 1998). Vibrio spp. are indigenous to seawater where they can exchange genes, including rRNA genes, by direct physical contact or indirectly through bacteriophages, known to be present in large amounts in seawater. This has been shown to be the case for Vibrio cholerae (for a review, see Wommack \& Colwell, 2000).

In this study polymorphism was explored in type strains and environmental isolates of the genus Vibrio by a method which allows easy testing of large numbers of strains. It consists of the observation of the formation of heteroduplexes after PCR amplification of the $16 \mathrm{~S}$ rDNAs (Espejo et al., 1998). When a sample containing several $16 \mathrm{~S}$ rDNAs is PCR-amplified, products differing in sequence can anneal, forming hybrids (heteroduplexes) which migrate slower than the homoduplex during PAGE. We show that $16 \mathrm{~S}$ rDNA polymorphism is a common phenomenon among type strains of validly described Vibrio species and of related strains isolated from oysters and coastal waters in Chile. Comparison of the sequences showed a high concentration of variable sites in particular regions of the molecule; 70-96 and 440-496 (E. coli numbering). The significance and origin of the observed polymorphism is discussed.

\section{METHODS}

Strains and culture conditions. Bacterial strains corresponded to Vibrio parahaemolyticus ATCC $17802^{\mathrm{T}}$, Vibrio vulnificus ATCC 27562, Vibrio alginolyticus ATCC 17749 and Vibrio cholerae (ISP NO 01) and were directly obtained from the respective culture collections. Environmental isolates were obtained from seawater samples collected from two points along the Chilean coast; Coloso and Horcon at latitudes $23^{\circ}$ $\mathrm{S}$ and $32^{\circ} \mathrm{S}$, respectively. The samples were filtered through Whatman No. 1 filter paper and the bacteria were subsequently collected in a $0.4 \mu \mathrm{m}$ pore size membrane (Millipore). The bacteria in the membrane were resuspended by vortexing in $2 \mathrm{ml}$ alkaline peptone water (APW). Thereafter, $100 \mu \mathrm{l}$ of the suspension was streaked on thiosulfate citrate-bile-sucrose agar (TCBS) plates containing $2 \% \mathrm{NaCl}$. The plates were incubated overnight at 37 and $17^{\circ} \mathrm{C}$ for $16-18 \mathrm{~h}$. Strains isolated from oysters were obtained from oyster homogenates prepared as described previously (Romero \& Espejo, 2002).
DNA extraction and hybridization. DNA for PCR was isolated from single bacterial colonies picked from marine agar plates, resuspended in $50 \mu \mathrm{l} \mathrm{TE}(0 \cdot 01 \mathrm{M}$ Tris, 0.001 M EDTA, pH 8.0) and lysed by boiling for $15 \mathrm{~min}$. The lysate was then centrifuged at $5000 \mathrm{~g}$ for $15 \mathrm{~s}$ and $1.5 \mu \mathrm{l}$ of the supernatants was directly used as template for amplification.

For Southern blotting, DNA of $V$. parahaemolyticus was purified as described by Sambrook et al. (1989). DNA $(3.5 \mu \mathrm{g})$ was then digested with 9 units EcoRI (Gibco-BRL), resolved on a $1.5 \%$ agarose gel and the Southern blot was performed on Hybond-N + membrane as described by the manufacturer (Boehringer Mannheim). A DNA probe of $1.4 \mathrm{~kb}$, specific for $16 \mathrm{~S}$ rDNA, was generated by PCR amplification of DNA extracted from V. parahaemolyticus and labelled by random priming with digoxigenin 11-dUTP using a DIG DNA labelling kit (Boehringer Mannheim) as recommended in the manufacturer's instructions. Hybridization and immunological detection of DIG-labelled probe were performed using a DIG-luminescence detection kit, according to the manufacturer's instructions (Boehringer Mannheim). Hybridization was performed using standard hybridization buffer at $68^{\circ} \mathrm{C}$ with washing as recommended by the manufacturer (Boehringer Mannheim).

PCR amplification of DNA, heteroduplex analysis, cloning and sequencing of the amplified 16S rDNA. 16S rDNA PCR amplification and the heteroduplex mobility assay were performed as described by Espejo et al. (1998), except that electrophoresis was conducted at $150 \mathrm{~V}$. PCR products were cloned into TOPO TA, according to the procedure indicated by the manufacturer (Invitrogen). Plasmid DNA was obtained by a rapid alkaline extraction miniprep (Birnboim, 1983). The DNA was diluted $(1: 500, \mathrm{v} / \mathrm{v})$ in sterile distilled water and $15 \mu \mathrm{l}$ was used for $16 \mathrm{~S} \mathrm{rDNA}$ amplification as described above. For sequencing, plasmids were purified with a Wizard Plus SV Minipreps rapid plasmid miniprep system (Promega). Cloned 16S rDNA genes were sequenced with an Applied Biosystems 310 automatic sequencer using ABI Prism dye terminator sequencing kits with M13 universal primers or primers annealing to conserved internal sequences. These primers were Eubac27F (5'-AGAGTTTGATCCTGGCTCAG-3'), 357F (5'-CTCCTACGGGAGGCAGCA-3'), 946F (5'-CCCGCACAAGCGGTGGA-3'), 1492R (5'-GGTTACCTTGTTACGACTT-3'), 1100R (5'-GGGTTGCGCTCGTTG-3') and 518R (5'-CGTATTACCGCGGCTGCTGG-3'). DNA sequences were inspected individually and manually assembled. The alignments and sequence similarities were performed with CLUSTAL w at the EMBL Outstation, European Bioinformatics Institute (EBI). Sequence similarities at specific sites were manually calculated. The secondary structure analysis was carried out with the MFOLD programs, using the sequence analysis software package (Genetics Computer Group, Madison, WI, USA).

\section{RESULTS}

\section{$16 \mathrm{~S}$ polymorphism observed by the formation of heteroduplexes after amplification of the 16S rDNAs of different Vibrio species}

When the $16 \mathrm{~S}$ rDNAs in the DNA extracted from cultures of V.alginolyticus, V. parahaemolyticus and V. vulnificus, obtained from single colonies, were PCRamplified, additional bands with slower than expected electrophoretic migration rates were observed after electrophoresis in polyacrylamide gels (Fig. 1a). As previously shown (Espejo et al., 1998), these bands 
(a)

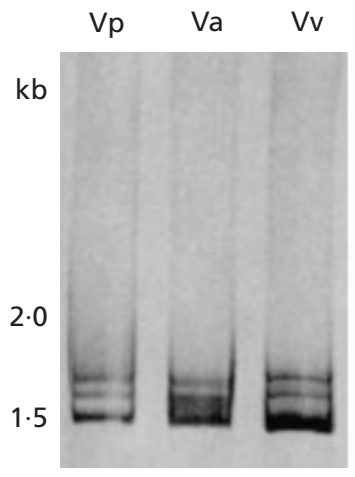

(b)

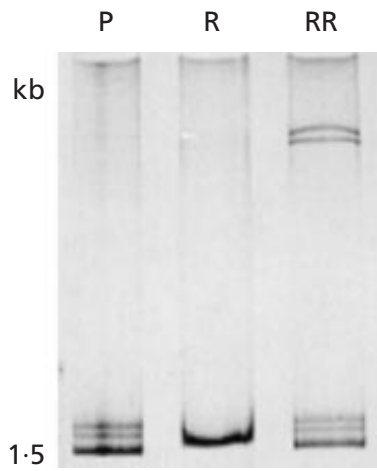

Fig. 1. (a) PAGE of $16 \mathrm{~S}$ rDNAs obtained after PCR amplification of DNA extracted from purified $V$. parahaemolyticus $(V p) V$. alginolyticus ( $\mathrm{Va}$ ) and $V$. vulnificus (Vv). (b) Heteroduplex nature of products observed above the $1.45 \mathrm{~kb}$ band after PAGE. Lanes: P, amplification products of $V$. parahaemolyticus; $\mathrm{R}$, products obtained after a single amplification cycle of samples in lane $\mathrm{P}$ diluted $1 / 10$ [this dilution was performed to prevent annealing between single-stranded products (five times the volume applied for electrophoresis in lane $\mathrm{P}$ was used to compensate for the dilution performed, assuming that the product was doubled after the amplification cycle)]; RR, products obtained after denaturation and renaturation of the samples applied in lane R. The position of molecular size markers is shown.

correspond to heteroduplexes formed between single strands of amplified $16 \mathrm{~S}$ rDNAs differing in nucleotide sequence. Single strands formed after the denaturation step of the amplification cycle can anneal during the temperature decrease to the annealing step. When the strands annealed are not $100 \%$ complementary, heteroduplexes are formed. Subjecting the product to an additional amplification cycle, after appropriate dilution to avoid annealing of the single strands (Delwart et al., 1993), can show their heteroduplex nature. In these conditions the heteroduplexes disappear. Furthermore, the presence of different sequences can be again revealed by the reappearance of the heteroduplexes if the latter product is subjected to denaturation and renaturation. Fig. 1(b) shows the results obtained after subjecting the heterogeneous amplification products of V. parahaemolyticus (shown in Fig. 1a) to this procedure. The same results were obtained with the amplification products of the other two species (not shown). These results suggest that some of the rrn operons in each of these species contained polymorphic $16 \mathrm{~S}$ rDNAs. Based on previous results relating the relative electrophoretic migration rate of the heteroduplexes to that of the homoduplex with sequence dissimilarity (Espejo et al., 1998), the sequence differences may be estimated to be about $2-3 \%$. If only the relative migration rate of the heteroduplexes formed by the Vibrio species is considered (Fig. 2), the equation can be restated as percentage dissimilarity $=-12.96 \times$ relative migration rate $+13 \cdot 03$. Using this equation, the intra-organism sequence dissimilarity observed in each of these strains can be estimated to be between 0.6 and $1 \cdot 1 \%$.

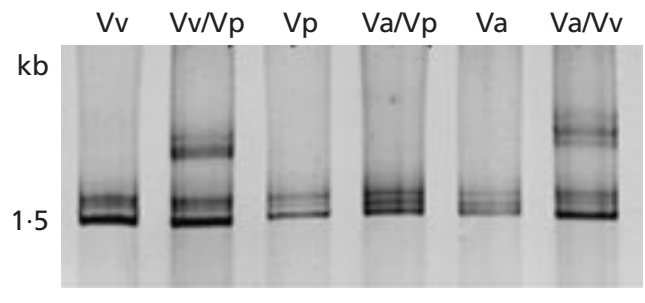

Fig. 2. $P A G E$ of $16 S$ rDNA obtained after $P C R$ amplification and of the products obtained after annealing of these $16 \mathrm{~S}$ rDNAs. Lanes $V v, V p$ and $V a$ show the amplification products of $V$. vulnificus, $V$. parahaemolyticus and $V$. alginolyticus, respectively. Lanes $\mathrm{Vv} / \mathrm{Vp}, \mathrm{Va} / \mathrm{Vp}$ and $\mathrm{Va} / \mathrm{Vv}$, show the products obtained after denaturation and renaturation between the amplification products of the corresponding pairs. (a)

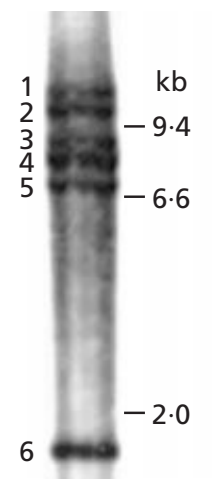

(b)

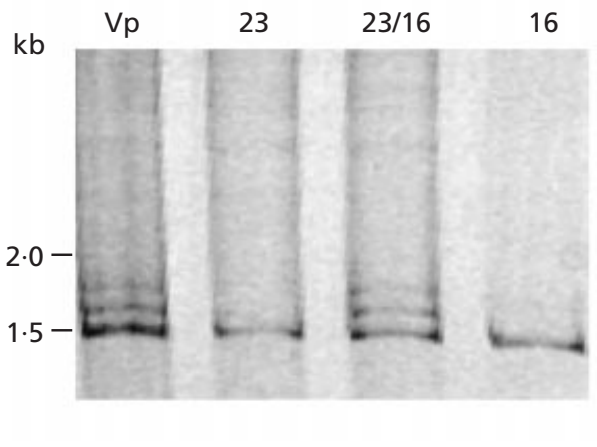

Fig. 3. Southern blot analysis and cloning of $r r n$ operons of $V$. parahaemolyticus. (a) Genomic DNA prepared from a culture of V. parahaemolyticus was cleaved with EcoRI, subjected to electrophoresis and then hybridized with a $16 \mathrm{~S}$ rDNA-specific probe prepared by PCR amplification and labelled for chemiluminescence. (b) Products of the PCR amplification of DNA from clones containing $16 \mathrm{~S}$ rDNAs of $V$. parahaemolyticus. Lanes: $V p$, amplification products of genomic DNA from $V$. parahaemolyticus; 23 , amplification product of plasmid DNA from clone 23; 16, amplification product of plasmid DNA from clone 16; $23 / 16$, products obtained after hybridization of the amplification products shown in lanes 23 and 16. Self-hybridization of products in lanes 23 and 16 did not change their patterns (not shown).

\section{Southern blot analysis, and cloning and sequencing of the 16S rDNA in different $r$ rn operons}

The above observations prompted us to further characterize the rrn operons of these Vibrio species. Southern blot hybridization of EcoRI-restricted V. parahaemolyticus DNA with a $16 \mathrm{~S}$ rDNA-specific probe yielded six bands, indicating the presence of at least six rrn operons in this genome (Fig. 3a). Hybridization between fragments and observation of heteroduplex formation indicated that fragments 5 and 6 contained 16S rDNA with different sequences (not shown). The amplification product of $V$. parahaemolyticus DNA was then cloned and the clones were distinguished according to the capacity of their amplified $16 \mathrm{~S}$ rDNA to form heterodu- 
Table 1. Comparison of $V$. parahaemolyticus (VP) $16 \mathrm{~S}$ rDNA amplified from different clones and from a restriction fragment containing 165 rDNA (F6)

$\mathrm{H}$ indicates the number of heteroduplexes observed between amplified 16S rDNA of the corresponding clones. $\mathrm{T}$ indicates the total number of differences found by comparison of 1514 sites. RV2 indicates the number of differences found in the 440-496 region (E. coli numbering). VP17802 ${ }^{\mathrm{T}}$ corresponds to the sequence reported for V. parahaemolyticus ATCC $17802^{\mathrm{T}}$. ND, Not done.

\begin{tabular}{|c|c|c|c|c|c|c|}
\hline Clone & & VP27 & F6 & VP44 & VP23 & VP17802 \\
\hline \multirow[t]{3}{*}{ VP16 } & $\mathrm{H}$ & 0 & 0 & 2 & 2 & ND \\
\hline & $\mathrm{T}$ & 4 & ND & 14 & 12 & 12 \\
\hline & RV2 & 0 & 0 & 11 & 10 & 7 \\
\hline \multirow[t]{3}{*}{ VP27 } & $\mathrm{H}$ & & ND & 2 & 2 & ND \\
\hline & $\mathrm{T}$ & & ND & 16 & 15 & 13 \\
\hline & RV2 & & 0 & 11 & 10 & 7 \\
\hline \multirow[t]{3}{*}{ F6 } & $\mathrm{H}$ & & & 2 & 2 & ND \\
\hline & $\mathrm{T}$ & & & ND & ND & ND \\
\hline & RV2 & & & 11 & 11 & 7 \\
\hline \multirow[t]{3}{*}{ VP44 } & $\mathrm{H}$ & & & & 0 & ND \\
\hline & $\mathrm{T}$ & & & & 5 & 19 \\
\hline & RV2 & & & & 1 & 12 \\
\hline \multirow[t]{3}{*}{ VP23 } & $\mathrm{H}$ & & & & & ND \\
\hline & $\mathrm{T}$ & & & & & 16 \\
\hline & RV2 & & & & & 12 \\
\hline
\end{tabular}

plexes, and the migration rate of the heteroduplex if formed. Hybridization between the $16 \mathrm{~S}$ rDNA amplified from two clones, $\mathrm{Vp} 23$ and $\mathrm{Vp} 16$, resulted in the formation of three bands, producing a similar pattern to that observed after amplification of the whole genome (Fig. 3b). The lowest and most intense band corresponds to the homoduplex, while the other two, appearing above this one, correspond to the reciprocal hybrids formed by each plus and minus complementary strand (Espejo et al., 1998). Although the extent of dissimilarity in this hybrid pair is identical, non-paired regions may form distinct structural conformations in each hybrid, decreasing the mobility to different extents (Jensen \& Straus, 1993). The potentiality of generating the three bands observed after amplification of the whole genome of $V$. parahaemolyticus with only two different $16 \mathrm{~S}$ rDNAs suggested that the polymorphism in this strain might consist of only two different $16 \mathrm{~S}$ rDNAs. The $16 \mathrm{~S}$ rDNAs amplified from the above-mentioned clones and from clones Vp44 and Vp27, which hybridized like clones Vp23 and Vp16, respectively, were sequenced. The amplification product of fragment 6, eluted from a parallel gel to that used for the Southern blot shown in Fig. 3(a), was also sequenced. Hybridization of $16 \mathrm{~S}$ rDNA amplified from this fragment rendered similar heteroduplexes to those obtained with the amplified products of clones 16 and 27. In every clone, the whole $16 \mathrm{~S}$ rRNA region between primers Eubac $27 \mathrm{f}$ and $1492 \mathrm{R}$ was sequenced. The main differences in sequence observed are summarized in Table 1 . From 4 up to 19 different nucleotide sites were found between the $16 \mathrm{~S}$ rDNAs of the analysed clones. Interestingly, $63-83 \%$ of these sites were concentrated in a stem-loop of the variable region between positions 440 and 496 (E. coli numbering), called RV2 in Table 1.

\section{5 rDNA polymorphism in environmental bacterial strains clustering among vibrios}

Bacterial isolates from oysters or seawater with $94 \%$ or higher similarity in $16 \mathrm{~S}$ rDNA to $V$. parahaemolyticus, $V$. cholerae and V. vulnificus were selected for this study. This value was chosen because the highest degree of dissimilarity between classical strains of the genus Vibrio is $7 \%$. The similarity was estimated by the relative migration rate of the $16 \mathrm{~S}$ rDNA heteroduplexes as shown in Fig. 2. 16S rDNA from the 20 strains selected by this criterion was amplified and examined for the formation of heteroduplexes, indicative of $16 \mathrm{~S}$ rDNA polymorphism. The amplification products of every strain examined showed the presence of additional bands, migrating immediately above the amplified $16 \mathrm{~S}$ rDNA, corresponding to heteroduplexes. At least three of these bands were observed in two strains, two bands in eight strains and one band in ten strains. Fig. 4 shows the heteroduplexes observed after amplification of $16 \mathrm{~S}$ rDNA from some of these strains. A few of the strains rendered heteroduplexes with slower relative migration rates than those observed in V. parahaemolyticus (see lanes $2 \mathrm{~d}$ and $3 \mathrm{~d}$, Fig. 4), suggesting the existence of up to $1.8 \%$ dissimilarity between $16 \mathrm{~S}$ rRNAs genes in these strains. Fig. 4 also compares the heteroduplexes generated by hybridization of the amplified $16 \mathrm{~S}$ rDNAs of these strains with those of $V$. parahaemolyticus. The heteroduplex nature of the bands observed in these isolates was confirmed by amplification after dilution 
(a)

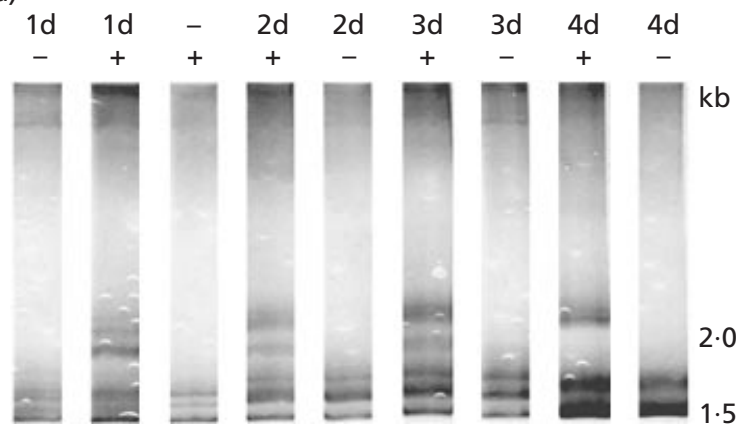

(b)

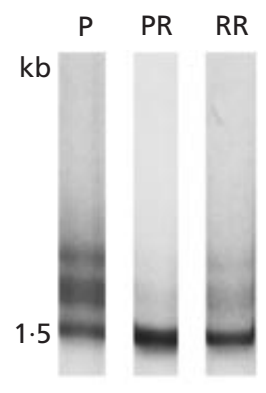

Fig. 4. (a) PAGE of 165 rDNA amplification products obtained from some environmental vibrio isolates and of the hybridization products of these amplification products with $V$. parahaemolyticus amplified $16 \mathrm{~S}$ rDNA. Lanes $1 \mathrm{~d} /-, 2 \mathrm{~d} /-, 3 \mathrm{~d} /-$ and $4 \mathrm{~d} /-$ contain the amplification product from the corresponding vibrio isolates. Lanes $1 \mathrm{~d} /+$, $2 \mathrm{~d} /+, 3 \mathrm{~d} /+$ and $4 \mathrm{~d} /+$ show the product of the hybridization between $16 \mathrm{~S}$ rDNA amplified from the corresponding strain with that from $V$. parahaemolyticus. (b) Heteroduplex nature of bands observed after amplification of DNA from strain 3d. Lanes: $P$, amplification products; $R$, products obtained after a single amplification cycle of samples in lane $P$; $R R$, products obtained after denaturation and renaturation of the samples applied in lane $\mathrm{R}$. The procedure was performed as described in the legend to Fig. 1(b). The position of molecular size markers is shown.

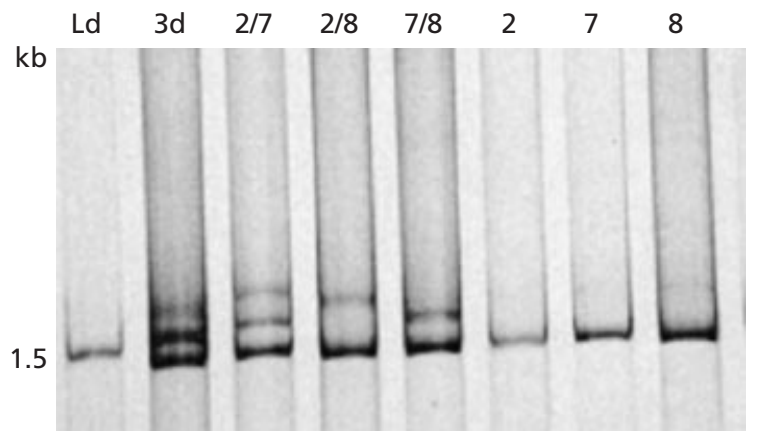

Fig. 5. Gel electrophoresis of the amplification products of different clones of $16 \mathrm{~S}$ rDNA of the environmental strain 3d and of the product obtained after hybridization of amplified 165 rDNA of the different clones. Lanes 3d, 2, 7 and 8 show the products obtained after amplification for 16S rDNA of strain 3d and clones 2, 7, 8 obtained with amplified 16S rDNA from strain $3 d$. Lanes $2 / 7,2 / 8$ and $7 / 8$ show the hybridization products between amplified 16S rDNA from the corresponding clones. Lane Ld shows the $1.5 \mathrm{~kb}$ molecular size marker.

and subsequent annealing, as described above for the type strains. Fig. 4(b) shows the results of this assay performed with strain $3 \mathrm{~d}$. Similar results were obtained with the other strains (not shown).

\section{Cloning and sequencing of the different 16S rDNAs in environmental isolate $\mathbf{3 d}$}

$16 \mathrm{~S}$ rDNA from isolate 3d was amplified and subsequently cloned. Clones containing different $16 \mathrm{~S}$ rDNAs were subsequently clustered according to the capacity of their amplified $16 \mathrm{~S}$ rDNA to form heteroduplexes. Fig. 5 shows the heteroduplexes formed between amplified $16 \mathrm{~S}$ rDNAs of clones clustered in different groups. Clones $3 \mathrm{~d} 2$ and $3 \mathrm{~d} 7$ rendered a pattern with two putative heteroduplexes (lane 2/7), similar in migration rate to those observed after amplification of the whole genomic DNA (lane 3d), suggesting that there were only two
Table 2. Comparison of $16 \mathrm{~S}$ rDNA amplified from different clones of Vibrio sp. 3d containing 16S rDNA

$\mathrm{H}$ indicates the number of heteroduplexes observed between amplified $16 \mathrm{~S}$ rDNA of the corresponding clones. $\mathrm{T}$ indicates the total number of differences found by comparison of 1514 sites. RV1 and RV2 indicate the number of differences found in the 70-96 and 440-496 regions (E. coli numbering), respectively.

\begin{tabular}{|llrrr|}
\hline Clone & & 3D8 & 3D4 & 3D7 \\
\hline \multirow{2}{*}{ 3D2 } & H & 1 & 1 & 2 \\
& T & 29 & 31 & 19 \\
& RV1 & 13 & 13 & 0 \\
& RV2 & 11 & 11 & 11 \\
3D8 & H & & 0 & 1 \\
& T & & 5 & 20 \\
& RV1 & & 0 & 13 \\
& RV2 & & 1 & 4 \\
& H 4 & & & 1 \\
& T & & & 29 \\
& RV1 & & & 13 \\
& RV2 & & & 3 \\
\hline
\end{tabular}

different $16 \mathrm{~S}$ rDNAs in this strain. However, hybridization of the product of each of these clones with that of a third clone, named $3 \mathrm{~d} 8$, produced only one putative heteroduplex band (lanes $2 / 8$ and 7/8, Fig. 5). Since clones $3 \mathrm{~d} 2,3 \mathrm{~d} 7$ and $3 \mathrm{~d} 8$ contain different $16 \mathrm{~S}$ rDNAs, it is likely that isolate $3 \mathrm{~d}$ contains at least three different $r r n$ operons. The nucleotide sequence was obtained from the amplified $16 \mathrm{~S}$ rDNA of clones $3 \mathrm{~d} 2,3 \mathrm{~d} 7$ and $3 \mathrm{~d} 8$, and also from clone $3 \mathrm{~d} 4$, which behaved like $3 \mathrm{~d} 8$ in heteroduplex analysis. A summary of the nucleotide differences is shown in Table 2. From 5 up to 31 different nucleotide sites were found. These sites were concentrated in the stem-loops containing nucleotides 70-96 
and 440-496 (E. coli numbering), called RV1 and RV2, respectively. Interestingly, the sequence of the RV1 region was identical in each clone of $V$. parahaemolyticus examined.

\section{DISCUSSION}

Detection of heteroduplexes after PCR amplification of DNA from purified bacterial strains proved to be a reliable indicator of the presence of polymorphism within particular organisms. This method allowed us to screen a large number of type strains and environmental isolates of the genus Vibrio and to show that polymorphism in $16 \mathrm{~S}$ rDNAs rrn operons of the same organism is a common phenomenon among bacterial species of this genus. The sequence heterogeneity estimated by the relative migration of the heteroduplexes was confirmed by nucleotide sequencing of cloned $16 \mathrm{~S}$ rDNAs from strain $3 \mathrm{~d}$ and $V$. parahaemolyticus. The absence of polymorphism in the amplification product of cloned $16 \mathrm{~S}$ rDNA, observed by either heteroduplex formation or sequencing, indicates that the reported polymorphism is not generated during PCR amplification.

The number of bands corresponding to heteroduplexes does not correspond to the number of genes differing in sequence. As shown earlier, reciprocal hybrids formed by each plus and minus complementary strand can display different migration rates; therefore two different $16 \mathrm{~S} \mathrm{rDNAs}$ can produce two heteroduplexes (Espejo et al., 1998). This is exemplified by the hybridization of the clones from strain $3 \mathrm{~d}$ where only two clones rendered two heteroduplexes with different electrophoretic migration. Formation of heteroduplexes with slower electrophoretic migration rates than the homoduplex corresponded with the number of mismatches in the variable region. The retarded electrophoretic migration rate observed for the heteroduplexes might be due to interruptions of the double helix by either singlestranded regions or stem-loops in the region in which the mismatches are concentrated.

Comparative analysis of the sequences obtained from the different clones of $V$. parahaemolyticus and isolate $3 \mathrm{~d}$ revealed details of the heterogeneity among $\mathrm{rrn}$ operons. Polymorphic nucleotide sites of the $16 \mathrm{~S}$ rRNA were concentrated in two hypervariable regions in strain 3d (RV1 and RV2), but only in one (RV2) in strain ATCC $17802^{\mathrm{T}}$ of $V$. parahaemolyticus. These two regions have been recognized as hypervariable regions when comparing $16 \mathrm{~S}$ rRNAs from different bacterial genera (Gray et al., 1984), as well as from different species of the genus Vibrio (Dorsch et al., 1992). However, grouping of polymorphic sites does not occur in every Vibrio species; the concentrations observed in these strains is not present in the 16S rRNA genes of the genome sequence of V. cholerae strain El Tor N16961 (Heidelberg et al., 2000). Concentrations of polymorphic sites may also occur in different regions; the stem region, designated helix 10, including nucleotides 180 200 (E. coli numbering), which is conserved in $V$. parahaemolyticus and strain 3d, has been shown to be highly polymorphic in Streptomyces strains (Ueda et al., 1999). The presence of short segments with a high number of base variations could be explained by recombination of short segments with laterally transferred rRNA genes (Wang \& Zhang, 2000). Most of the substitutions observed in this region show compensating base covariation, allowing preservation of the secondary structure and probably also of the function. The accumulation of so many compensating changes implies that the divergence of the different versions of this stem-loop is relatively ancient. The presence in the same bacteria of molecules that diverged a long time ago may be explained in two ways: by conservation, because this polymorphism provides a selective advantage, or by acquisition of a version evolved in other bacteria. In our opinion both explanations are attractive. On the one hand, the possibility that the high polymorphism is due to lateral transfer is interesting, as it would suggest that this phenomenon is common in marine Vibrio species. High rates of horizontal transfer of rRNA genes is increasingly accepted. It has been shown that this transfer is not precluded by the co-evolution of rRNA with many other components in the translational machinery, as previously assumed (see, for example, Lawrence, 1999; Asai et al., 1999). It has been suggested that the different $16 \mathrm{~S}$ rDNA found in Thermomonospora chromogena was acquired from Thermobispora bispora or a related organism via horizontal gene transfer (Yap et al., 1999). Similarly, it has been postulated that the rrnE region of Salmonella subspecies I may correspond to that of E. coli (Perez et al., 1998). On the other hand, it is conceivable that having polymorphic $16 \mathrm{~S}$ rDNAs may constitute a selective advantage to the bacteria. For example, it could imply the possibility of having several $16 \mathrm{~S}$ rRNA molecules that might respond differently to the presence of compounds which interact with bacterial ribosomes, such as certain antibiotics. A review of the sites in bacterial $16 \mathrm{~S}$ rRNA associated with antibiotic resistance (Hu \& Ochi, 2001; Recht et al., 1999) showed no correspondence with the RV1 and RV2 sites observed in strain $3 \mathrm{~d}$ and V.parahaemolyticus. However, it has been shown that nucleotide sites 76-90 and 456-476 in the $16 \mathrm{~S}$ rRNA of $E$. coli are required for specific interaction with protein S4, a ribosomal protein essential for ribosome assembly (Sapag et al., 1990). These regions correspond in part to the variable regions RV1 and RV2.

\section{ACKNOWLEDGEMENTS}

It is a pleasure to acknowledge the support, help and advice of M. García-Varela and J. P. Laclette for nucleotide sequencing in their laboratory at the Instituto de Investigaciones Biomédicas of the Universidad Nacional Autónoma de México. We would also like to thank Illani Atwater for reviewing the manuscript and correcting the English grammar and style. This work was supported by grants FONDECYT 1990765 and FONDECYT 2000064 from the Comisión Nacional de Investigación Científica y Tecnologica, Chile. C. Moreno acknowledges a scholarship from Deutscher Akademischer Austausch Diemst (DAAD). 


\section{REFERENCES}

Amann, R. I., Ludwig, W. \& Schleifer, K. H. (1995). Phylogenetic identification and in situ detection of individual microbial cells without cultivation. Microbiol Rev 59, 143-169.

Asai, T., Zaporojets, D., Squires, C. \& Squires, C. L. (1999). An Escherichia coli strain with all chromosomal rRNA operons inactivated: Complete exchange of rRNA genes between bacteria. Proc Natl Acad Sci US A 96, 1971-1976.

Birnboim, D. (1983). A rapid alkaline extraction method for the isolation of plasmid DNA. Methods Enzymol 100, 243-255.

Cilia, V., Laffay, B. \& Christen, R. (1996). Sequence heterogeneities among $16 \mathrm{~S}$ ribosomal RNA sequences, and their effect on phylogenetic analysis at the species level. Mol Biol Evol 13, 451-461.

Dahllof, I., Baillie, H. \& Kjelleberg, S. (2000). $r p o B$-based microbial community analysis avoids limitations inherent in $16 \mathrm{~S}$ rRNA gene intraspecies heterogeneity. Appl Environ Microbiol 66, 33763380

Delwart, E. L., Shpaer, E. G., Louwagie, J., McCutchan, F. E., Grez, M., Rübsamen-Waigman, H. \& Mullins, J. I. (1993). Genetic relationships determined by a DNA heteroduplex mobility assay: analysis of HIV-1 env genes. Science 262, 1257-1261.

Dorsch, M., Lane, D. \& Stackebrandt, E. (1992). Towards a phylogeny of the genus Vibrio based on $16 \mathrm{~S}$ rRNA sequences. Int J Syst Bacteriol 42, 58-63.

Espejo, R. T., Feijóo, C. G., Romero, J. \& Vásquez, M. (1998). PAGE analysis of the heteroduplexes formed between PCRamplified $16 \mathrm{~S}$ rRNA genes: estimation of sequence similarity and rDNA complexity. Microbiology 144, 1611-1617.

Fegatella, F., Lim, J., Kjelleberg, S. \& Cavicchioli, R. (1998). Implications of rRNA operon copy number and ribosome content in the marine oligotrophic ultramicrobacterium Sphingomonas sp. strain RB2256. Appl Environ Microbiol 64, 4433-4438.

Felsenstein, J. (1985). Confidence limits on phylogenies: an approach using the bootstrap. Evolution 39, 783-791.

Gray, M. W., Sankoff, K. \& Cedergren, R. J. (1984). On the evolutionary descent of organisms and organelles: A global phylogeny based on a highly conserved structural core in small subunit ribosomal RNA. Nucleic Acids Res 12, 5837-5852.

Heidelberg, J. F., Eisen, J. A., Nelson, W. C. \& 29 other authors (2000). DNA sequence of both chromosomes of the cholera pathogen Vibrio cholerae. Nature 406, 477-483.

Hu, H. \& Ochi, K. (2001). Novel approach for improving the productivity of antibiotic-producing strains by inducing combined resistant mutations. Appl Environ Microbiol 67, 1885-1892.

Jensen, M. A. \& Straus, N. (1993). Effect of PCR conditions on the formation of heteroduplex and single-stranded DNA products in the amplification of bacterial ribosomal DNA spacer regions. PCR Methods Appl 3, 186-194.

Lamfrom, H., Sarabhai, A. \& Abelson, J. (1978). Cloning of Beneckea genes in Escherichia coli. J Bacteriol 133, 354-363.

Lawrence, J. G. (1999). Gene transfer, speciation, and the evolution of bacterial genomes. Curr Opin Microbiol 2, 519-523.

Nubel, U., Engelen, B., Felske, A., Snaidr, J., Wieshuber, A., Amann, R. I., Ludwig, W. \& Backhaus, H. (1996). Sequence heterogeneities of genes encoding $16 \mathrm{~S}$ rRNAs in Paenibacillus polymyxa detected by temperature gradient gel electrophoresis. $J$ Bacteriol 178, 5636-5643.

Perez, S., Rodriguez, F., Lan, R. \& Reeves, P. R. (1998). Variation of the ribosomal operon $16 \mathrm{~S}-23 \mathrm{~S}$ gene spacer region in representatives of Salmonella enterica subspecies. J Bacteriol 180, 2144-2151.

Recht, M. I., Douthwaite, S. \& Puglisi, J. D. (1999). Basis for prokaryotic specificity of action of aminoglycoside antibiotics. EMBO J 18, 3133-3138.

Reischl, U., Feldmann, K., Naumann, L., Gaugler, B. J., Ninet, B. \& Hirschel, B. (1998). $16 \mathrm{~S}$ rRNA sequence diversity in Mycobacterium celatum strains caused by presence of two different copies of $16 \mathrm{~S}$ rRNA gene. J Clin Microbiol 36, 1761-1764.

Romero, J. \& Espejo, R. T. (2002). The prevalence of non-cultivable bacteria in oysters (Tiostrea chilensis, Philippi 1845). J Shellfish Res (in press).

Ruimy, R., Breittmayer, V., Elbaze, P., Lafay, B., Bouseemart, O., Gauthier, M. \& Christen, R. (1994). Phylogenetic analysis and assessment of the genera Vibrio, Photobacterium, Aeromonas, and Plesiomonas deduced from small-subunit rRNA sequences. Int J Syst Bacteriol 44, 416-426.

Sambrook, J., Fritsch, E. F. \& Maniatis, T. (1989). Molecular Cloning: a Laboratory Manual, 2nd edn. Cold Spring Harbor, NY : Cold Spring Harbor Laboratory.

Sapag, A., Vartikar, J. \& Draper, D. (1990). Dissection of the $16 \mathrm{~S}$ rRNA binding site for ribosomal protein S4. Biochim Biophys Acta 1050, 34-37.

Ueda, K., Seki, T., Kudo, T., Yoshida, T. \& Kataoka, M. (1999). Two distinct mechanisms cause heterogeneity of $16 \mathrm{~S}$ rRNA. $J$ Bacteriol 181, 78-82.

Wang, Y. \& Zhang, Z. (2000). Comparative sequence analyses reveal frequent occurrence of short segments containing an abnormally high number of non-random base variations in bacterial rRNA genes. Microbiology 146, 2845-2854.

Wang, Y., Zhang, Z. S. \& Ramanan, N. (1997). The actinomycete Thermobispora bispora contains two distinct types of transcriptionally active $16 \mathrm{~S}$ rRNA genes. J Bacteriol 179, 3270-3276.

Wolfe, C. J. \& Haywood, M. G. (1993). Bioluminescent symbionts of the Caribbean flashlight fish (Kryptophanaron alfredi) have a single rRNA operon. Mol Mar Biol Biotechnol 2, 189-197.

Wommack, K. E. \& Colwell, R. R. (2000). Viroplankton : viruses in aquatic ecosystems. Microbiol Mol Biol Rev 64, 69-114.

Yamaichi, Y., lida, T., Park, K.-S., Yamamoto, K. \& Honda, T. (1999). Physical and genetic map of the genome of Vibrio parahaemolyticus: presence of two chromosomes in Vibrio species. Mol Microbiol 31, 1513-1521.

Yap, W. H., Zhang, Z. \& Wang, Y. (1999). Distinct types of rRNA operons exist in the genome of the actinomycete Thermomonospora chromogena and evidence for horizontal transfer of an entire rRNA operon. J Bacteriol 181, 5201-5209.

Received 2 October 2001; revised 11 December 2001; accepted 17 December 2001. 